\title{
IMPLICANCIA FINANCIERA DE LA \\ ACTUALIZACIÓN DE INTERESES POR EL PAGO EXTEMPORÁNEO DE LA DEUDA TRIBUTARIA
}

\author{
FINANCIAL IMPLICATIONS OF INTEREST BY UPDATING LATE \\ PAYMENT OF TAX DEBT
}

Adolfo Valencia Gutiérrez*

Docente Asociado de la Facultad de Ciencias Contables - UNMSM

[Recepción: Julio de 2012/ Conformidad: Agosto 2012]

\section{RESUMEN}

El nacimiento del tributo que se configura por la dación de una Ley o un Decreto Legislativo en caso de delegación de facultades considerados taxativamente en el artículo $74^{\circ}$ de la Constitución Política del Perú, dentro de las técnicas tributarias también se tipifica claramente el nacimiento de la obligación tributaria configurado en el Artículo $2^{\circ}$ del Código Tributario lo cual indica: la obligación tributaria nace cuando se realiza el hecho previsto en la ley, como generador de dicha obligación, el cual es de derecho público y es el vínculo entre el acreedor y el deudor tributario, establecido por ley, que tiene por objeto el cumplimiento de la prestación tributaria, siendo exigible coactivamente según el artículo $1^{\circ}$ del Código Tributario.

Es por ello que una vez que nace la obligación tributaria solamente queda el hecho de ser satisfecha el cumplimiento de la prestación tributaria, sean estas sustanciales o formales y el incumplimiento oportuno de la prestación dineraria pueden generar intereses contemplados en la normatividad tributaria lo cual tienen implicancias financieras para el contribuyente, claro está que por el pago extemporáneo del mismo el Estado está en la obligación de exigir el pago de intereses como una forma de resarcimiento por el bien cautelado.

Palabras Clave: Tributo, obligación tributaria, tasa de interés moratorio, tributo insoluto, días transcurridos.

\begin{abstract}
The birth of a tax that is set by the enactment of a law or a legislative decree in case of delegation considered exhaustively in Article 74 of the Political Constitution of Peru, within the technical tax which also clearly typifies the birth of the obligation tax set out in Article 2 of the Tax Code states: the tax liability arises when performing the action described in the law, as a generator of the this obligation, which is public law and is the link between the creditor and the debtor, established by law, which aims at providing tax compliance, being coercively enforceable under Article 1 of the Tax Code. That is why once the tax liability arise these is only the fact of being satisfied with the compliance tax provision, whether substantive or formal, and failure of timely payment of money can generate interest referred to in tax regulations which have financial implications for the taxpayer, it is clear that by the late payment of the same, the state is obliged to demand interest payments as a form of compensation for the good provided.
\end{abstract}

Keywords: Tribute, tax liability, interest rate on arrears, unpaid tax, days elapsed.

* Doctor en Ciencias de la Educación, y Magister en Ciencias de la Educación con mención en Docencia Universitaria. UNE. Contador Público Colegiado. UNMSM, E-mail: avalenciag@unmsm.edu.pe 


\section{INTRODUCCIÓN}

La obligación tributaria, que es de derecho público, es el vínculo entre el acreedor y el deudor tributario, establecido por ley, que tiene por objeto el cumplimiento de la prestación tributaria, siendo exigible coactivamente. Debemos tener presente que la Obligación es un vínculo de naturaleza jurídica, y la "prestación" es el contenido de la obligación, esto es, la conducta del deudor tributario. En este sentido, la conducta o prestación del deudor tributario es el "pagar la deuda tributaria", la finalidad o el objeto de la obligación es que el deudor pague la deuda tributaria, y si no lo hace, la Administración se encuentra facultada para exigirle coactivamente o forzosamente el cumplimiento de la misma.

La configuración del hecho imponible (aspecto material), su conexión con una persona, con un sujeto (aspecto personal), su localización en determinado lugar (aspecto espacial) y su consumación en un momento real y fáctico determinado (aspecto temporal), determinarán el efecto jurídico deseado por la ley, que es el nacimiento de una obligación jurídica concreta, a cago de un sujeto determinado, en un momento también determinado. ${ }^{1}$

El Código Tributario Peruano establece que: "La obligación tributaria nace cuando se realiza el hecho previsto en la Ley, como generador de dicha obligación" (TUO Cód. Tributario Art. 2)

\section{Concepto de deuda tributaria}

La deuda tributaria, en general, "está constituida por la prestación o conjunto de prestaciones pecuniarias a que un sujeto pasivo resulta obligado frente a la Hacienda Pública en virtud de distintas situaciones jurídicas derivadas de la aplicación de tributos" Como sabemos, el término genérico tributo comprende:

Impuesto: Es el tributo cuyo cumplimiento no origina una contraprestación directa a favor del contribuyente por parte del Estado.

Contribución: es el tributo cuya obligación tiene como hecho generar beneficios derivados de la realización de obras públicas o de actividades estatales.

Tasa: es el tributo cuya obligación tiene como hecho generador la prestación efectiva por el estado de un servicio público individualizado en el contribuyente.

Por otro lado, previamente a definir la multa tributaria, veamos el significado de infracción tributaria: "toda acción u omisión que importe violación de las normas tributarias, constituye infracción sancionable de acuerdo con las normas establecidas en el código tributario"3.

En este sentido, en relación a la aplicación de sanciones, se ha establecido que la Administración Tributaria aplica por la comisión de "infracciones", las sanciones consistentes en multa, comiso, internamiento temporal de vehículos, cierre temporal de establecimiento $\mathrm{u}$ oficina de profesionales independientes y suspensión de licencias, permisos, concesiones o autorizaciones vigentes otorgadas por entidades del Estado para el desempeño de actividades o servicios públicos.

1 http://blog.pucp.edu.pe/item/18396/introduccion-a-la-obligacion-tributaria

2 HUAMANI, Rosendo. Código tributario comentado. Juristas Editores EIRL, 2011, p.355

3 VALENCIA, Adolfo. Glosario Tributario. Fondo Editorial San Marcos, febrero 2011, p.71 
En este orden de ideas, la multa es una sanción que impone la administración tributaria por la comisión (entendida como acción u omisión) de una infracción tributaria.

Por su parte, los intereses comprenden: i) el interés moratorio por el pago extemporáneo del tributo Art. 33 Cód. Tributario; ii) el interés moratorio aplicable a las multas impagas; iii) el interés por aplazamiento y/o aplazamiento de pago previsto.

La deuda tributaria es el resultado de sumar una serie de valores en dinero que el sujeto pasivo de la obligación tributaria ha de pagar por diversos conceptos: esto es el tributo, recargos, multa, interés moratorio.

Si bien se puede considerar directamente como deuda tributaria el objeto de la prestación tributaria (el tributo en sí) su contenido legal es más amplio pues no sólo considera como deuda al indicado objeto de la prestación tributaria, sino incluye a las multas y a los intereses. Como tal, la deuda tributaria es unitaria y está constituida por la suma o monto que el deudor debe o adeuda (por tributo o cuota más los intereses, y, si hubiere, las multas) al acreedor tributario, y cuyo pago total será exigido por la Administración Tributaria. Talledo Mazú, en esa línea, sostiene que la deuda tributaria es la "suma adeudada al acreedor tributario por tributos, multas, el interés moratorio, el interés de fraccionamiento o aplazamiento"

De acuerdo con el Art. 28 del Código Tributario (CT) la deuda tributaria está constituida por el tributo, la multa y los intereses moratorios, es decir, la deuda tributaria es la suma adeudada al acreedor tributario por tributos, multa e intereses.

Los elementos de la deuda tributaria son: i) el elemento principal o capital, es el componente básico de toda deuda. Sin la presencia de este elemento no podrán originarse los elementos accesorios o intereses. Depen- diendo del tipo de obligación tributaria que origine la deuda (sean obligaciones sustanciales u obligaciones formales), el elemento principal que la conforma podrá ser el tributo o la multa; y ii) los elementos accesorios o intereses, la legislación actual contempla a los intereses como elemento accesorio de la deuda, sin embargo, esto no siempre fue así, debido a que hasta hace algunos años, se consideraba también como elementos accesorios a los recargos y reajustes.

\section{Concepto de actualización de deuda}

El legislador ha optado en el artículo 74 de la Constitución vigente por la clasificación tripartita, en este sentido, cuando el artículo 28 del CT señala "tributo", la referencia puede ir a Impuesto, Contribución o Tasa. Por otro lado, cuando se señala a la multa, el legislador se está refiriendo a un tipo de sanción, que se aplica como consecuencia de la comisión de una infracción.

En relación a los intereses, el legislador tributario utiliza los dos tipos de interés que existen teniendo en cuenta su origen (legal y convencional), el interés legal, que nace y se devenga porque la ley así lo ha previsto, y donde la voluntad de las partes es completamente ajena. $\mathrm{Y}$ en relación a la función que cumple ese interés (compensatorio o moratorio), ha decidido por el interés moratorio, que tiene por finalidad indemnizar la mora en el cumplimiento de una prestación dineraria y por el interés compensatorio.

En este sentido, es que este artículo precisa que el interés puede ser: i) el interés moratorio por el pago extemporáneo del tributo; este interés tiene origen legal, y se devenga automáticamente a partir del día siguiente al vencimiento del plazo otorgado por el legislador para el pago del tributo. Asimismo, se indica que a este interés se le denomina TIM (Tasa de Interés Moratorio) a que se refiere 
el artículo 33 del CT; ii) el interés moratorio por el pago extemporáneo de las multas impagas; éste es el mismo interés (TIM) que se aplica a los tributos impagos referidos anteriormente; y iii) el interés por aplazamiento y/o fraccionamiento de pago previsto en el artículo $36^{\circ}$.

Para nuestro Código Tributario, la mora en el cumplimiento del pago de la deuda tributaria no constituye una infracción, ya que tal como se establece en el artículo 33 del CT: "El monto del tributo no pagado dentro de los plazos indicados en el Código devengará un interés equivalente a la TIM". En tal sentido, en nuestro sistema tributario se aplica la mora automática, por lo que, el no pago oportuno de la deuda tributaria, trae como consecuencia inmediata el devengamiento de intereses, pero no constituye una infracción.

Por consiguiente la actualización de la deuda tributaria implica la determinación del adeudo tributario hasta la fecha de pago, considerando previamente: i) el tributo o principal insoluto, ii) los días transcurridos y iii) la tasa de interés vigente al nacimiento de la obligación tributaria.

\section{Tipos de intereses}

Interés, según Tamames y Gallego, es la cantidad que se paga como remuneración por la disponibilidad de una suma de dinero tomada en concepto de crédito.

Los intereses considerados componentes de la deuda tributaria (como prestaciones accesorias de la obligación tributaria) de acuerdo a nuestro Código Tributario, son: i) los intereses moratorios, ii) los intereses por aplazamiento $\mathrm{y} / \mathrm{o}$ fraccionamiento (que no son intereses moratorios) $)^{4}$.

Podemos considerar que el interés compensatorio constituye la contraprestación 4 Huamaní, op. cit. p. 357 por el uso de un dinero o de cualquier otro bien, cuyo valor se exprese en dinero. Se origina en un acuerdo entre el acreedor y el deudor, si bien es cierto que en este acuerdo las partes pueden fijar libremente la tasa de interés a aplicar, deben respetar los límites máximos de interés permitidos por las normas. A esto se le conoce como interés legal.

El interés moratorio tiene como finalidad resarcir o indemnizar la mora o retardo en el pago. El deudor tiene que indemnizar al estado por el retraso, viéndose obligado a pagar un monto adicional a la deuda original, con el objeto de resarcir el daño ocasionado al acreedor, sin distinguir su intencionalidad.

Los intereses que se generan desde el nacimiento de la obligación hasta su fecha de vencimiento, siempre tienen el carácter de interés compensatorio. Luego del vencimiento, cualquier interés generado tendrá la calidad de interés moratorio.

La naturaleza del interés moratorio tributario no difiere en esencia del considerado en el Derecho privado; así, podemos conceptuarlo como señala el Código Civil (artículo 1242): el interés es moratorio cuando tiene por finalidad indemnizar la mora en el pago.

El presupuesto de hecho de esta prestación no es otro que el retraso en el pago de la obligación tributaria. Los casos que dan lugar al interés de demora se remiten a un retraso en el ingreso de la prestación con respecto a los plazos en que debería haberse efectuado (plazos establecidos genéricamente, para nuestro caso, por los artículos 3 y 29 del Código Tributario).

Estos intereses tienen naturaleza indemnizatoria (de los daños, o perjuicios causados por ese retraso), resarciendo al acreedor tributario y evitando el enriquecimiento injusto del deudor que dispone de unas sumas de dinero debidas más allá del tiempo en que había 
de cumplir su prestación; por tanto, no cabe apreciar en el interés por demora un componente punitivo (sancionador) ni siquiera disuasorio, lo que explica su procedencia con independencia de que el retraso en el pago obedezca a actuaciones lícitas o ilícitas.

Así tenemos, de acuerdo con lo regulado en el artículo 33, al interés moratorio por el pago extemporáneo del tributo y a los intereses en los anticipos y pagos a cuenta según el artículo 34, indicando igualmente, aun cuando no está vinculado, a la obligación tributaria; en el rubro de intereses también tenemos al interés moratorio aplicable a las multas (consideradas como deuda tributaria), regulado por el artículo 181 del Código Tributario. La Tasa de Interés Moratorio (TIM) es la alícuota que se cobra como interés sobre la deuda tributaria insoluta, esto es, sobre el tributo o multa dejado de pagar. De conformidad con el primer párrafo del artículo 33 del Código Tributario, la Tasa de Interés Moratorio no deberá exceder del $10 \%$ por encima de la tasa activa del mercado promedio mensual en moneda nacional (TAMN) que publique la SBS el último día hábil del mes anterior.

El TIM mensual es fijado por la Administración Tributaria; la TIM diaria resulta de dividir la TIM mensual entre 30 días, el resultado debe ser en 5 decimales; la TIM acumulada resulta de multiplicar "tasa de interés diario por el número de días", lo cual debe ser en 3 decimales. ${ }^{5}$

\section{VARIACIÓN DE LA TASA DE INTERES MORATORIO (TIM)}

\begin{tabular}{|c|c|c|c|c|c|}
\hline \multirow{2}{*}{$\begin{array}{c}\text { Fecha de } \\
\text { publicación }\end{array}$} & Base Legal & \multicolumn{2}{|c|}{ Vigencia } & \multicolumn{2}{c|}{ Tasa de Interés Mora- } \\
te & & \multicolumn{2}{c|}{ torio } \\
\hline 02-12-1992 & R.S No.214-92-EF/SUNAT & $01-12-1992$ & $30-04-1993$ & 7.6 & 0.23333 \\
\hline $30-04-1993$ & R.S No.50-1993-F/SUNAT & $01-05-1993$ & $31-07-1993$ & 6.0 & 0.20 \\
\hline $30-07-1993$ & R.S No.085-93-EF/SUNAT & $01-06-1993$ & $30-09-1993$ & 5.5 & 0.18333 \\
\hline $05-10-1993$ & R.S No.101-93-EF/SUNAT & $01-10-1993$ & $31-12-1993$ & 4.5 & 0.15 \\
\hline $04-01-1994$ & R.S No.001-94-EF/SUNAT & $21-01-1994$ & $30-04-1994$ & 4.0 & 0.13333 \\
\hline 28-04-1994 & R.S No.43-94-EF/SUNAT & $01-05-1994$ & $30-06-1994$ & 3.5 & 0.11666 \\
\hline $01-07-1994$ & R.S No.54-94-EF/SUNAT & $01-07-1994$ & $30-09-1994$ & 3.0 & 0.10 \\
\hline $30-09-1994$ & R.S No.79-94-EF/SUNAT & $01-10-1994$ & $02-02-1996$ & 2.50 & 0.08333 \\
\hline $02-02-1996$ & R.S No.11-96-EF/SUNAT & $03-02-1996$ & $31-12-2000$ & 2.20 & 0.07333 \\
\hline $30-12-2000$ & R.S No.144-2000-EF/SUNAT & $01-01-2001$ & $31-10-2001$ & 1.80 & 0.06 \\
\hline $31-10-2001$ & R.S No.126-2001-EF/SUNAT & $01-02-2003$ & $06-02-2003$ & 1.60 & 0.05333 \\
\hline $06-02-2003$ & R.S No.32-2003-EF/SUNAT & $07-02-2003$ & $31-12-2004$ & 1.50 & 0.05 \\
\hline 26-12-2004 & Ordenanza No.108-2004-MPC & $01-01-2005$ & $03-04-2007$ & 1.00 & 0.03333 \\
\hline 03-04-2007 & $\begin{array}{c}\text { Ordenanza 008-007-MPCTIM } \\
\text { SUNAT }\end{array}$ & $04-04-2007$ & $28-02-2010$ & 1.50 & 0.05 \\
\hline 17-02-2010 & $\begin{array}{c}\text { R.S No.53-2010/SUNAT Orde- } \\
\text { nanza No.008-2007-MPC }\end{array}$ & $01-03-2010$ & Continúa & 1.20 & 0.04 \\
\hline
\end{tabular}




\section{Actualización general de la deuda tribu taria}

El cálculo del interés moratorio está regulado por el tercer párrafo del Artículo 33 del Código Tributario, lo cual fue modificado referente a la capitalización de intereses, ya que anteriormente se capitalizaba los intereses al $31 / 12$ de cada año, es decir al principal (tributo) se capitalizaba el accesorio (intereses actualizados al 31 de diciembre de cada año), la sumatoria se constituía como el nuevo principal para los siguientes períodos, lo cual constituía un problema económico para el

\section{ACTUALIZACIÓN GENERAL DE LA DEUDA TRIBUTARIA}

\begin{tabular}{|c|c|c|c|c|}
\hline Fecha de inicio & $\rightarrow$ & 31 de Diciembre & & Fecha de pago \\
\hline Tributo o multa & Corren intereses & Capitalización & $\begin{array}{c}\text { Corren tributos } \\
\text { mas intereses }\end{array}$ & $\begin{array}{l}\text { Último día de la } \\
\text { actualización }\end{array}$ \\
\hline \multicolumn{5}{|c|}{ Las formulas aplicables a la actualización de la deuda son: } \\
\hline \multicolumn{5}{|c|}{ T.I.M. } \\
\hline \multicolumn{5}{|c|}{ TIM diaria vigente = ---------------------- } \\
\hline \multicolumn{5}{|c|}{$\begin{array}{l}\text { Interés diario= TIM diaria x No de días x Mto. Trib. o multa } \\
\text { TE: solamente se capitaliza hasta el } 31 / 12 / 2005 \text { posterior a esa fecha se calcula } \\
\text { intereses corridos }\end{array}$} \\
\hline
\end{tabular}

contribuyente por la onerosidad de las deudas capitalizadas, con la dación del D. Leg $\mathrm{N}^{\circ} 969$ publicado el 24-12-06 que entró en vigencia el 25-12-06. La modificación al artículo 33, elimina la capitalización de intereses en el cálculo de los intereses moratorios por deudas tributarias no pagadas en los plazos establecidos. $^{6}$

De esta manera, para establecer el monto de la deuda tributaria no pagada oportunamente, bastará con determinar los intereses moratorios diarios sin capitalizarlos al $31 \mathrm{de}$ diciembre de cada año. En ese sentido, para calcular el interés moratorio acumulado se deberá establecer el número de días transcurridos entre el vencimiento de la obligación y la fecha de pago de la deuda tributaria y multiplicarse por la tasa de interés moratoria diaria. Sobre el particular, se debe tener en claro que la no capitalización de intereses opera únicamente para los intereses moratorios que se hubieran devengado a partir de la vigencia de la norma modificatoria; de tal manera que, una deuda tributaria correspondiente al período enero 2005 o ejercicios anteriores cuyo pago se efectué en enero de 2011 deberá ser capitalizada al 31 de diciembre de 2005 pero no al 31 de diciembre del 2011. A partir del 2006 solamente se considera los intereses por los días transcurridos hasta la fecha de pago inclusive. 
IMPLICANCIA FINANCIERA DE LA ACTUALIZACIÓN DE INTERESES POR

EL PAGO EXTEMPORANEO DE LA DEUDA TRIBUTARIA

\section{Casos prácticos de actualización de la deuda tributaria}

\subsection{ACTUALIZACIÓN DE LA DEUDA TRIBUTARIA SIN CAPITALIZACIÓN DE INTE RESES}

Factura de Venta

\begin{tabular}{|l|l|l|}
\hline V.V. & & $531,564,102.19$ \\
\hline$(-)$ DSCTO. & $15 \%$ & $-\mathbf{7 9 , 7 3 4 , 6 1 5 . 3 3}$ \\
\hline (+) INTERES & $2 \%$ & $10,631,282.04$ \\
\hline (+) FLETE & & $10,500.00$ \\
\hline $\begin{array}{l}\text { BASE IMPO- } \\
\text { NIBLE }\end{array}$ & & $462,471,268.91$ \\
\hline IGV & $19 \%$ & $87,869,541.09$ \\
\hline P. DE VENTA & & $550,340,810.00$ \\
\hline
\end{tabular}

Factura de Compra

\begin{tabular}{|l|l|l|}
\hline V.C. & & $210,339,613.25$ \\
\hline$(-)$ DSCTO. & $13 \%$ & $\mathbf{- 2 7 , 3 4 4 , 1 4 9 . 7 2}$ \\
\hline (+) INTERES & $1 \%$ & $2,103,396.13$ \\
\hline (+) FLETE & & $15,300.00$ \\
\hline $\begin{array}{l}\text { BASE IMPONI- } \\
\text { BLE }\end{array}$ & & $185,114,159.66$ \\
\hline IGV & $19 \%$ & $35,171,690.34$ \\
\hline P. DE COMPRA & & $220,285,850.00$ \\
\hline
\end{tabular}

\section{OPERACIONES SOBRE VENTAS}

a) Base Imponible-Flete $=$ Valor de venta $462^{\prime} 471,268.91-10,500=462^{\prime} 460,768.91$

b) Valor de venta $=100 \%$ Descuento $=(15 \%)$ Intereses $\frac{20 \%}{87 \%} \mathrm{~V} . \mathrm{V}$

c) Para determinar el V. de venta $=\underline{462^{\prime} 460,768.91 \times 100}$

d) VV Determinado $=531$ '564,102.19

\section{OPERACIONES SOBRE COMPRAS}

a) Base Imponible-Flete $=$ Valor de compra $185^{\prime} 144,159.66-15,300=185^{\prime} 098,859.66$

b) Valor de compra $=100 \%$

Descuento $=(13 \%)$

Intereses $\quad 1 \%$ V.C $88 \%$

c) Para determinar el V. de compra $=\underline{185^{\prime} 098,859.66 \times 100}$

d) VC Determinado $=210 ’ 339,613.25$

\section{DETERMINACIÓN DE LA OBLIGACIÓN TRIBUTARIA}

\begin{tabular}{|l|c|}
\hline & \multicolumn{2}{|c|}{ IGV } \\
\hline IGV VENTA & $87,869,541.09$ \\
\hline IGV COMPRA & $-35,171,690.34$ \\
\hline IGV A PAGAR & $\mathbf{5 2 , 6 9 7 , 8 5 0 . 7 6}$ \\
\hline
\end{tabular}

\begin{tabular}{|l|c|}
\hline \multicolumn{2}{|c|}{ I.RENTA } \\
\hline VENTAS & $462,471,268.91$ \\
\hline COEFICIENTE & $2 \%$ \\
\hline I.RA PAGAR & $\mathbf{9 , 2 4 9 , 4 2 5 . 3 8}$ \\
\hline
\end{tabular}


ACTUALIZACIÓN DE LA DEUDA TRIBUTARIA

ACTUALIZACIÓN DE LA DEUDA TRIBUTARIA AL 22/11/2011

\section{Calendario del 2009 al 2011}

\begin{tabular}{|c|c|c|c|c|c|c|c|}
\hline Meses Años & 2007 & 2008 & 2009 & 2010 & 2011 & \multicolumn{2}{|c|}{ TOTAL DIAS A ACTUALIZAR } \\
\hline Enero & & & 0 & 31 & 31 & DIAS AÑO 2000 & 0 \\
\hline Febrero & & & 0 & 28 & 28 & DIASAÑO 2001 & 0 \\
\hline Marzo & & & 0 & 31 & 31 & DIAS AÑO 2002 & 0 \\
\hline Abril & & & 8 & 30 & 30 & DIAS AÑO 2003 & 0 \\
\hline Mayo & & & 31 & 31 & 31 & DIASAÑO 2004 & 0 \\
\hline Junio & & & 30 & 30 & 30 & DIAS AÑO 2005 & 0 \\
\hline Julio & & & 31 & 31 & 31 & DIAS AÑO 2006 & 0 \\
\hline Agosto & & & 31 & 31 & 31 & DIASAÑO 2007 & 0 \\
\hline Septiembre & & & 30 & 30 & 30 & DIAS AÑO 2008 & 0 \\
\hline Octubre & & & 31 & 31 & 31 & DIASAÑO 2009 & 253 \\
\hline Noviembre & & & 30 & 30 & 22 & DIAS AÑO 2010 & 365 \\
\hline Diciembre & & & 31 & 31 & 0 & DIAS AÑO 2011 & 326 \\
\hline TOTAL DÍAS & 0 & 0 & 253 & 365 & 326 & TOTAL DIAS & 944 \\
\hline
\end{tabular}

VIGENCIA DE LAS TASAS DE INTERES MORATORIO

\begin{tabular}{|c|c|c|c|c|}
\hline \multicolumn{5}{|c|}{ CÁLCULO DE INTERES DIARIO } \\
\hline VIGENCIA & TIM & DIAS & $\%$ & $\begin{array}{l}\text { INTERES } \\
\text { DIARIO }\end{array}$ \\
\hline $07 / 02 / 2003$ & 1.5 & 30 & 100 & 0.0005 \\
\hline $01 / 03 / 2010$ & 1.2 & 30 & 100 & 0.0004 \\
\hline
\end{tabular}

\begin{tabular}{|c|c|c|c|c|}
\hline \multicolumn{5}{|c|}{ INTERES MORATORIO } \\
\hline \multicolumn{5}{|c|}{ CÁLCULO DEL TRIBUTO ACTUALIZADO AL: 31/12/2009 } \\
\hline IGV A PAGAR & $\begin{array}{c}\text { DIAS } \\
\text { TRANSCURRIDOS }\end{array}$ & $\begin{array}{c}\text { INTERES DIARIO } \\
\%\end{array}$ & $\begin{array}{c}\text { TOTAL } \\
\text { INTERÉS }\end{array}$ & DEUDA CAPITALIZABLE \\
\hline $52,697,850.76$ & 253 & 0.0005 & $6,666,278.12$ & $59,364,128.88$ \\
\hline
\end{tabular}

\begin{tabular}{|l|r|r|r|l|}
\hline CONCEPTO & AL: $28 / 02 / 2010$ & $\begin{array}{l}\text { AL: } \\
\text { 31/12/2010 }\end{array}$ & AL: 22/11/2011 & AL: \\
\hline IMPORTE & $52,697,850.76$ & $52,697,850.76$ & $52,697,850.76$ & \\
\hline DIASTRANSCURIDOS & 59 & 306 & 326 & \\
\hline INTERES DIARIO & 0.0005 & 0.0004 & 0.0004 & \\
\hline TOTAL & $\mathbf{1 , 5 5 4 , 5 8 6 . 6 0}$ & $\mathbf{6 , 4 5 0 , 2 1 6 . 9 3}$ & $\mathbf{6 , 8 7 1 , 7 9 9 . 7 4}$ & \\
\hline
\end{tabular}

TOTAL DEUDA TRIBUTARIA:

\begin{tabular}{|ll|c|}
\hline DEUDA AL: $31 / 12 / 2009$ & $59,364,128.88$ \\
\hline DEUDA AL: $28 / 02 / 2010$ & $1,554,586.60$ \\
\hline DEUDA AL: $31 / 12 / 2010$ & $6,450,216.93$ \\
\hline DEUDA AL: $22 / 11 / 2011$ & $6,871,799.74$ \\
\hline & \\
\hline & & \\
TOTALDEUDA & $\mathbf{7 4 , 2 4 0 , 7 3 2 . 1 5}$ \\
\hline
\end{tabular}

208/ QUIPURAMAYOC | Vol. 20(37) 2012 


\begin{tabular}{|l|c|l|c|}
\hline \multicolumn{1}{|c|}{ VENTAS } & \multicolumn{2}{c|}{ COMPRAS } \\
\hline Valor de venta & $22,059,008.31$ & Valor de Compra & $17,175,529.42$ \\
\hline Descuento (14\%) & $(3,088,261.16)$ & Descuento $(\mathbf{1 3} \%)$ & $(2,232,818.82)$ \\
\hline Interés 2\% & $441,180.17$ & Interés $2 \%$ & $343,510.59$ \\
\hline Flete & $\mathbf{3 8 . 2 2 0}$ & Flete & $\mathbf{2 3 . 4 6 0}$ \\
\hline Sub- Total & $19,450,147.31$ & Sub - Total & $15,309,681.18$ \\
\hline I.G.V. 19\% & $3,695,527,99$ & I.G.V. 19\% & $2,908,839,42$ \\
\hline Precio de Venta & $\mathbf{2 3 , 1 4 5 , 6 7 5 . 3 0}$ & Precio de Compra & $\mathbf{1 8 , 2 1 8 , 5 2 0 . 6 0}$ \\
\hline
\end{tabular}

1) $\mathrm{Sb}(\mathrm{PV})$

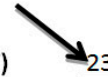

$145,675.30 \times 100$

119

$\mathrm{Sb}(\mathrm{PV})$

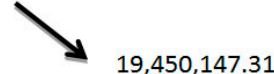

2) Subtotal-Flete $=19,576,759.07$

$19,411,927.31 \times 88 \%=22,059,008.31$

$22,059,008.31 \times 14 \%=3,088,261.16$

$22,059,008.31 \times 2 \%=441,180.17$
1) $\mathrm{Sb}(\mathrm{PC}) \triangle \frac{18,218,520.60 \times 100}{119}$

119

$\mathrm{Sb}(\mathrm{PC})$

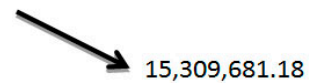

2) Subtotal - Flete $=15,286,221.18$

$15,286,221.18 \times 89 \%=17,175,529.42$

$17,175,529.42 \times 13 \%=2,232,818.82$

$17,175,529.42 \times 2 \%=343,510.59$

\section{Impuestos por Pagar}

\begin{tabular}{|c|c|c|c|}
\hline I.G.V. Ventas & I.G.V. Compras & I.G.V. por Pagar & $\begin{array}{c}\text { Impuesto a la } \\
\text { Renta }\end{array}$ \\
\hline $3,695,527,99$ & $2,908,839,42$ & $786,688.57$ & $389,002.95$ \\
\hline
\end{tabular}

I.G.V. Ventas - I.G.V. Compras = I.G.V. $x$ Pagar

$3,695,527.99-2,908,839.42=786,688.57$

\begin{tabular}{|cc|}
\hline $2 \%$ & $19,450,147.31$ \\
Impuesto a la Renta & $389,002.95$ \\
\hline
\end{tabular}

Mlarzo: $31-1 /=14$ marzo

Año $2004=290$ días

Año $2005=365$ días

Año $2006=365$ días

Año $2007=365$ días

Año $2008=366$ días

Año $2009=365$ días

Año $2010=59$ días

Año $2010=306$ días

Año $2011=365$ días $389,002.95$

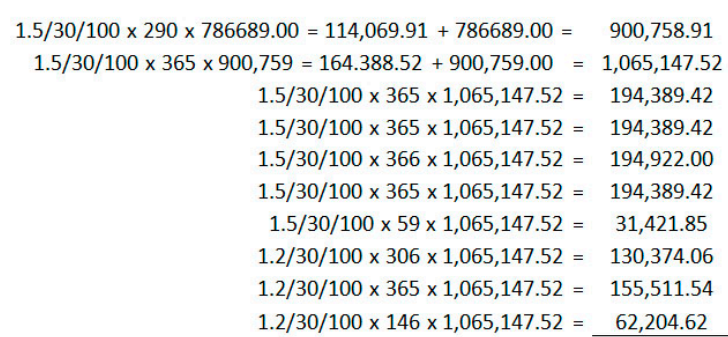

Adeudo Tributario $\quad 2,222,749.85$

* Los Tributos Afectos son : Impuesto a la Renta: $\quad 389,002.95$

I.G.V. : $786,688.57$ 


\section{CONTRASTACIÓN CON LA CALCULADORA TRIBUTARIA : SUNAT}

6.1 Primer caso, actualización de la deuda tributaria.

\begin{tabular}{|c|c|}
\hline \multicolumn{2}{|c|}{ Actualización de la Deuda } \\
\hline \multicolumn{2}{|c|}{$\begin{array}{l}\text { Para el siguiente cáalculo no se considera al cronograma de vencimiento especial de los Buenos Contribuyente } \\
\text { Para el tributo Renta de 3ra. Categoria, el cálculo del pago es a cuenta mensual }\end{array}$} \\
\hline \multicolumn{2}{|c|}{ Datos Ingresados de la deuda a actualizar: } \\
\hline Concepto de DEUDA & Deuda por declaración jurada \\
\hline RUC: & 20258505213 COMERCIO \& CIA S.A \\
\hline Tributo a actualizar & IGV - DPER. INT. - CTA. PROPIA \\
\hline Periodo Tributario & 200903 \\
\hline Importe del Tributo & $52,697,851$ \\
\hline Fecha hasta la que se atualizará & 22/11/2011 \\
\hline \multicolumn{2}{|r|}{ Pagos realizados } \\
\hline \multicolumn{2}{|r|}{ Importe pagado } \\
\hline \multicolumn{2}{|r|}{ Pagos aplicados al cálculo(*) } \\
\hline Fecha de $p$ & Importe pagado \\
\hline \multicolumn{2}{|l|}{ Resultado: } \\
\hline \multicolumn{2}{|c|}{ Fecha de vencimiento según RUC: $\quad 22 / 04 / 2009$} \\
\hline Importe del Tributo: & $52,697,851$ \\
\hline Saldo de la deuda tributaria a la fecha: & 22/11/2011 \\
\hline Total deuda calculada(*): & $74,240,732$ \\
\hline
\end{tabular}

6.2 Segundo caso, capitalización y actualización de la deuda tributaria

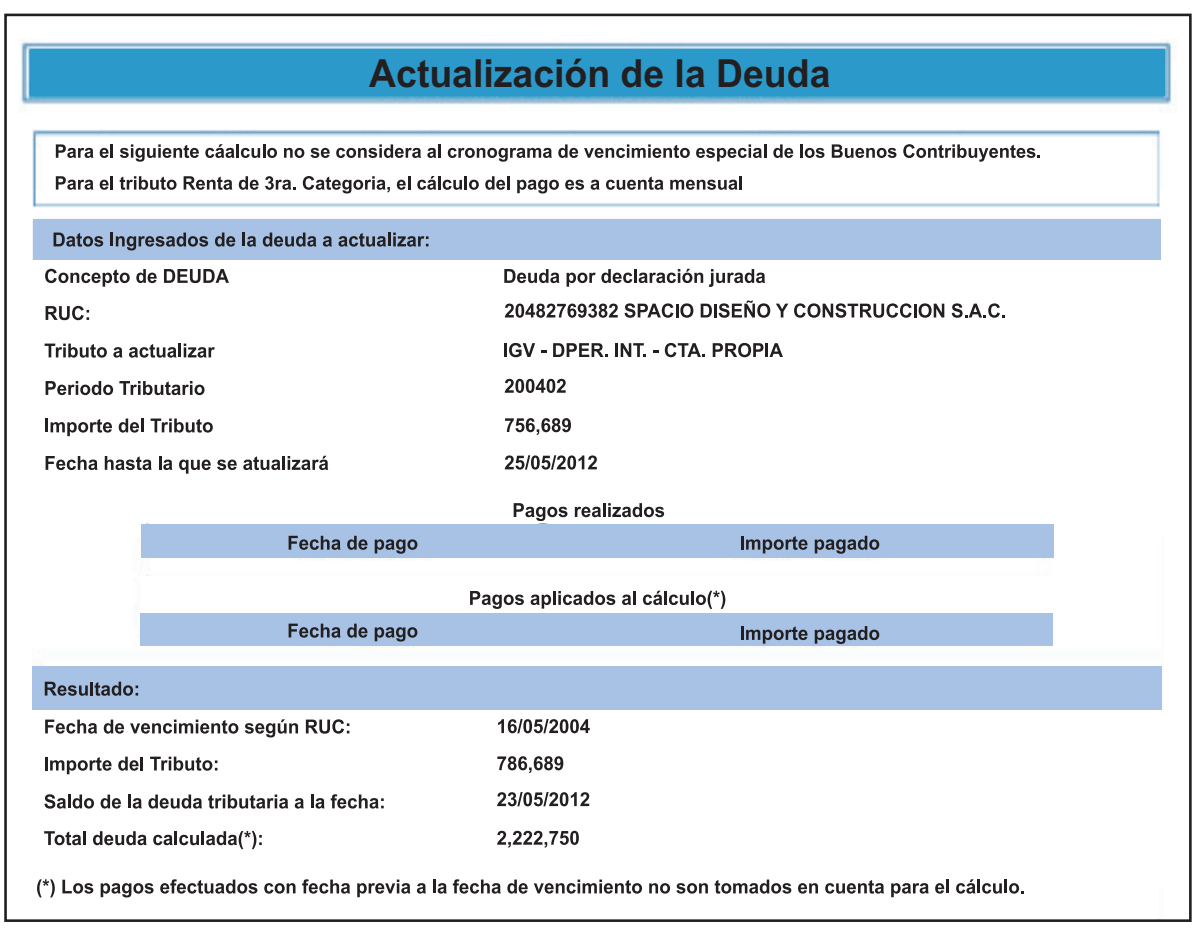




\section{CONCLUSIONES}

- Es necesario internalizar que el cumplimiento oportuno de la obligación tributaria por parte de los deudores tributarios, permite que el acreedor tributario, Estado, pueda contar con los recursos financieros para que pueda cumplir adecuada y oportunamente con su responsabilidad que los son inherentes.

- Es importante que el deudor tributario se informe correctamente de cuales son los componentes de la deuda tributaria, a fin de no incurrir en inobservancias del mismo y no estar involucrado en infracciones tributarias tipificadas taxativamente en el Código Tributario.

- Los deudores tributarios igualmente deben conocer, aplicar y cumplir en su oportunidad sus obligaciones tributarias sean estas formales o sustanciales, ya que el incumplimiento genera el nacimiento de la exigibilidad de la obligación a partir del día siguiente del vencimiento.

- Se debe entender que la tasa de interés moratorio es aplicado según nuestro Código Tributario como la forma de resarcir el daño causado al Estado por el incumplimiento oportuno de la prestación tributaria que le es exigible al contribuyente.

- La onerosidad por el incumplimiento oportuno de la obligación tributaria, genera implicancias financieras para el contribuyente que pueden generar muchas dificultades económicas.

\section{REFERENCIAS BIBLIOGRÁFICAS:}

1. Constitución Política del Perú, 1993

2. Texto único ordenado del Código Tributario, D.S No. 135-88-EF3. http://www.china. org.cu/spanish/xi-diafang/ningxia.html

3. Valencia Gutiérrez, Adolfo. 2011. Glosario tributario. Fondo Editorial. UNMSM.

4. Valencia Gutiérrez, Adolfo. 2011. Código Tributario. Fondo Editorial Inadep, octubre 2011, p.35

5. Carrasco Buleje, Rojas. 2007. Deuda Tributaria. Editorial Santa Rosa.

6. Huamaní Cueva, Rosendo. 2011. Código tributario comentado. Juristas Editores EIRL, Lima.

7. http://blog.pucp.edu.pe/item/70441/ una-aproximacion-al-concepto-de-tributo $(16-01-2012)$

8. http://blog.pucp.edu.pe/item/18396/ introduccion-a-la-obligacion-tributaria $(16 / 01 / 2012)$

9. http://www.sunat.gob.pe/legislacion/ nor_graf/mef/2008/ds-024-2008.pdf

10.http: / / orientacion.sunat.gob.pe/ index.php?option=com_conten t \&view $=$ article $\&$ id $=642:$ cas os \&catid=98: actualizacion-deuda-tributaria-con-ipc\&Itemid=167 (24/07/2012) 